\title{
AUTONOMÍA, CONFORMIDAD Y REBELIÓN: MOVIMIENTOS Y CULTURAS JUVENILES EN ALEMANIA EN EL PERIODO DE ENTREGUERRAS
}

\author{
ELIZABETH HARVEY \\ Universidad de Nottingham (Reino Unido)
}

RESUMEN: Este artículo examina las continuidades y discontinuidades entre los movimientos $y$ las culturas juveniles de la República de Weimar y los de la era nazi. En la Alemania de Weimar, un variado espectro de organizaciones juveniles proporcionaba a millones de jóvenes oportunidades de ocio junto con la posibilidad de luchar por intereses e ideas comunes en el marco de un grupo de edad. Después de 1933, el régimen nazi destruyó el pluralismo de la cultura juvenil de Weimar a la vez que buscó adoptar algunas características de los movimientos juveniles anteriores a 1933 en su campaña para imponer conformidad en la generación más joven en su conjunto. A su vez, esta campaña provocó diferentes respuestas desafiantes de la gente joven que buscaba subvertir la Juventud Hitleriana, evadirse de ella y afirmar su autonomía y que recurría para ello a elementos de la cultura juvenil anterior a 1933.

Palabras Clave: Alemania. Juventud. Movimientos juveniles. República de Weimar. Nacionalsocialismo.

\begin{abstract}
This article explores continuities and discontinuities between youth movements and youth cultures of the Weimar period and those during the Nazi era. A diverse spectrum of youth organizations in Weimar Germany provided millions of young people with leisure opportunities along with the chance to pursue common interests and ideas in a peer group setting. After 1933, the Nazi regime destroyed the pluralism of Weimar youth culture while seeking to adopt some features of pre-1933 youth movements in its drive to enforce conformity on an entire younger generation. In turn, this drive provoked a range of defiant responses from young people who sought to subvert and evade the Hitler Youth and assert their autonomy by drawing on elements of pre-1933 youth culture.
\end{abstract}

KEY WORDS: Germany. Youth. Youth movements. Weimar Republic. National Socialism. 
En las semanas que precedieron a las elecciones al Reichstag de julio de 1932, en las que los nacionalsocialistas resultaron el partido más importante, una serie de artículos titulados «Las organizaciones juveniles en la crisis actual» aparecieron en el periódico editado por el Consejo Nacional de Organizaciones Juveniles (Reichsausschuss der deutschen Jugendverbände) ${ }^{1}$. Dirigentes y representantes juveniles, principalmente hombres, de una gran variedad de organizaciones y ligas (Bünde) de movimientos juveniles comentaban los problemas a que hacían frente sus organizaciones en un periodo de profunda crisis económica, desempleo masivo y creciente radicalismo político. Mensajes contradictorios, esperanzadores y preocupados, surgían de esta colección de opiniones. Algunos declaraban que la «emergencia nacional» creaba nuevas oportunidades para el trabajo entre la juventud. Argumentaban que las formas de actividad de grupo desarrolladas dentro de las organizaciones juveniles podían ser aplicadas en proyectos para los desempleados; que la disciplina, el compromiso con un objetivo común y el liderazgo responsable característicos del movimiento juvenil podían ser un modelo para superar la fragmentación en la esfera política en general; que los cuadros de los movimientos juveniles existentes podían incluso llegar a ser los futuros dirigentes nacionales ${ }^{2}$. Frente a este optimismo provocador, las preocupaciones eran también evidentes. Un portavoz de los jóvenes trabajadores de oficinas destacaba la amenaza de radicalización política juvenil, mientras afirmaba que su organización, parte de la Confederación General de Trabajadores Alemanes (Allgemeiner Deutscher-Gewerkschaftsbund, ADGB), afiliada al Partido Socialdemócrata Alemán (Sozialdemokratische Partei Deutschlands, SPD), era capaz de hacer frente por igual a los izquierdistas radicales y a los nazis ${ }^{3}$. Un representante de los jóvenes trabajadores protestantes se preguntaba si no debía elevarse la edad mínima de voto establecida en los 20 años ${ }^{4}$. Hablando en nombre de la Federación de la Juventud Judía, Georg Lubinski advertía sobre el creciente antisemitismo que dañaba las posibilidades de los jóvenes judíos. Una portavoz de la Asociación Central de Jóvenes Católicas (Zentralverband der katholischen Jungfrauenvereinigungen Deutschlands) avisaba sobre el poder del «se-

1 «Sonderheft: Jugendverbände in der Krise der Gegenwart, Erste Folge», en Das Junge Deutschland (Berlín), vol. 26, $\mathrm{n}^{\circ} .5$ (mayo de 1932), pp. 125-154; «Sonderheft: Jugendverbände in der Krise der Gegenwart, Zweite Folge», en Das Junge Deutschland (Berlín), vol. 26, nº. 6 (junio de 1932), pp. 165-187; «Sonderheft: Jugendverbände in der Krise der Gegenwart: Dritte Folge», en Das Junge Deutschland (Berlín), vol. 26, nº. 7 (julio de 1932), pp. 197-216.

2 «Sonderheft: Jugendverbände in der Krise der Gegenwart, Erste Folge», op. cit., pp. 126133: aportaciones de Wolfgang Wieckberg, Bund der Wandervögel und Kronacher, Alfred Moritz, Freischar Junger Nation, y un representante del Deutsche Freischar.

3 «Sonderheft: Jugendverbände in der Krise der Gegenwart, Zweite Folge», op. cit., p. 167: colaboración de Ludwig Diederich, ZdA-Jugend.

4 «Sonderheft: Jugendverbände in der Krise der Gegenwart, Dritte Folge», op. cit., p. 197: artículo de Ernst Rudoph, Evangelische Arbeiterjugend.

5 Ibid., pp. 209-211: contribución de Georg Lubinski, Reichsausschuss der jüdischen Jugendverbände. 
guidismo» en la política y la fascinación por un líder seguro de la victoria. Ella predijo que «llegaría un momento cuando, como si de una ley de la naturaleza se tratara, un grupo bastante específico de caracteres más débiles y menos confiados en sí mismos, sucumbiría a la psicosis del vencedor (Siegerpsychose) y se uniría a la procesión de los millones» ${ }^{6}$.

Algunos temas comunes de este foro de opinión reflejaban ampliamente las visiones compartidas de ese momento. Por una parte, se consideraba que el impacto político y social de la depresión había creado una «cuestión juvenil» de nuevas dimensiones, expulsando del trabajo en el punto álgido de la crisis a más de un millón de jóvenes de entre 14 y 25 años, los límites de edad utilizados a menudo para definir a la «juventud». En julio de 1932, 1,46 millones de jóvenes entre 14 y 25 años estaban registrados como desempleados 7 . El desempleo masivo amenazaba con crear una «generación perdida» de jóvenes excluidos del mercado de trabajo y de la perspectiva de una edad adulta independiente $^{8}$. Se predecía que el descontento se podría añadir a lo que ya se había diagnosticado como la alienación de los jóvenes — tanto hombres como mujeres- del sistema democrático parlamentario, y que los hombres jóvenes predispuestos a la violencia podrían ser reclutados fácilmente por las organizaciones paramilitares de los partidos antirrepublicanos extremistas. Con el desarrollo demográfico alemán generando un número record de jóvenes votantes en torno a los 20 años, la conducta política de los jóvenes era una causa de preocupación y especulación aún mayor porque el papel de la edad en la conducta electoral era adivinado más que medido9. Como respuesta a esta situación, los dirigentes juveniles, particularmente los intelectuales procedentes del milieu de la Bündische Jugend ${ }^{10}$, afirmaban que la generación más joven debía

6 «Sonderheft: Jugendverbände in der Krise der Gegenwart, Erste Folge», op. cit., p. 152: colaboración de Else Peerenboom, Zentralverband der katholischen Jungfrauenvereinigungen.

7 HARVEY, Elizabeth: Youth and the Welfare State in Weimar Germany, Oxford, 1993, p. 107.

8 Peukert, Detlev: «The Lost Generation: Youth Unemployment at the End of the Weimar Republic», en Evans, Richard J. y GEARY, Dick (dirs.): The German Unemployed: Experiences and Consequences of Mass Unemployment from the Weimar Republic to the Third Reich, Londres, 1987.

9 ReULECKE, Jürgen: «...und sie werden nicht mehr frei ihr ganzes Leben!»: Der Weg in die «Staatsjugend» von der Weimarer Republik zur NS-Zeit», en HERRMANN, Ulrich y OELKERS, Jürgen (dirs.): Pädagogik und Nationalsozialismus, Weinheim y Basel, 1988, pp. 243-255, p. 245. Existen pocas evidencias concluyentes sobre la conducta de los jóvenes votantes en las elecciones al Reichstag durante la República de Weimar. Véase una discusión de los datos disponibles y de los problemas de analizar la conducta de voto por edades en FALTER, Jürgen: «The National Socialist Mobilization of New Voters: 1928-1933», en CHILDERS, Thomas (dir.): The Formation of the Nazi Constituency 1919-1933, Londres y Sydney, 1981, 202-231.

10 Bündische Jugend es un término colectivo usado para referirse a un conjunto de grupos diversos y más o menos grandes que surgieron después de la Primera Guerra Mundial en Alemania y que florecieron durante la República de Weimar. La falta de organización y de criterios formales para definir un grupo particular como bündisch hace que las estimaciones sobre el número y tamaño de estos grupos, que frecuentemente surgían, se dividían y cambiaban de nombre, sean aproximadas. 
contribuir a determinar la situación que sucediera al caos político y económico de la depresión ${ }^{11}$. Sin embargo, no eran sólo los dirigentes de la juventud los que estaban cautivados por la idea de una «misión de la generación más joven». Los políticos, tanto los que formaban parte del gobierno como los que no, se sentían atraídos por medidas que prometían entregarles el apoyo de la «joven generación», sondeaban a las organizaciones juveniles, y, generalmente, buscaban hacer que su movimiento o su programa pareciera más «juvenil»12. En 1932, por tanto, la «juventud» se construía como un problema y una causa potencial de desorden pero, al mismo tiempo, las visiones competitivas del futuro político de Alemania la consideraban como la potencial vanguardia de un nuevo orden que podía surgir.

Una estimación de 1924 sugiere que había alrededor de 1.000 grupos que podían ser considerados como parte de la Bündische Jugend: algunos tenían sólo unas pocas docenas de miembros, otros se unirían más adelante en organizaciones más grandes. Entre las Bünde más importantes en los últimos años de la República de Weimar estaban la Deutsche Freischar, la Grossdeutscher Jugendbund y la Jungnationaler Bund. Aunque el número total de miembros de las bünde probablemente nunca excedió de los 100.000 y no igualó a los de las organizaciones juveniles de masas católicas y protestantes, ejercieron una influencia en la cultura juvenil de su tiempo desproporcionada con respecto a su fuerza numérica. Eran una continuación pero también una evolución del movimiento excursionista (Wandervögel) anterior a la Primera Guerra Mundial y se distinguían de otras organizaciones juveniles de su tiempo por una serie de características distintivas. Un concepto clave era la consideración de la Bund como una comunidad dedicada a la autoeducación de sus miembros. Las ligas enfatizaban su autonomía: cada Bund hacía lo que le daba la gana, y cultivaba su identidad y «estilo» particular, permaneciendo apartada de los partidos políticos y otras instituciones para adultos. Sus miembros estaban unidos por la lealtad a un líder carismático elegido, que —al menos en teoría- era también joven. Además, normalmente nadie se convertía en miembro de una Bund sólo por su propia decisión, sino que, en general, era invitado a unirse a ella a partir de la percepción de «afinidades» con ésta. Las actividades de la Bund estaban segregadas por sexos, y la cohesión interna se fomentaba a través de actividades de ocio características como el excursionismo y el campismo, los debates de grupo y la composición de música. Sus socios eran jóvenes procedentes principalmente de las clases medias y que eran todavía estudiantes a tiempo completo. Estaban unidos por lazos de amistad y por un conjunto de valores comunes, más que por un programa o por objetivos políticos específicos. Pero las generalizaciones sobre la política de la Bündische Jugend son difíciles por la diversidad de las Bünde y la imprecisión del lenguaje que utilizaban para expresar sus opiniones sociales y políticas. Sin embargo, a pesar de su insistencia general en su postura «apolítica», las diferentes Bünde podían promover $-\mathrm{y}$ realmente lo hicieron - tendencias particulares entre sus miembros: en algunos casos dirigidas hacia el compromiso social y, en otros, hacia el ultranacionalismo y el autoritarismo.

11 Trommler, Frank: «Mission ohne Ziel. Über den Kult der Jugend im modernen Deutschland», en Koebner, Thomas, JanZ, Rolf-Peter, y Trommler, Frank (dirs.): «Mit uns zieht die neue Zeit»: Der Mythos Jugend, Frankfurt am Main, 1985, pp. 14-49, esp. pp. 40-45.

12 JONES, Larry Eugene: «Liberalism and the Challenge of the Younger Generation: The Young Liberal Struggle for a Reform of the Weimar Party System, 1928-30», en KRABBE, Wolfgang R. (dir.): Politische Jugend in der Weimarer Republik, Bochum, 1993, pp. 106-128; sobre los intentos de «rejuvenecer» la imagen del Partido Nacional Popular Alemán (el Deutschnationale Volkspartei (DNVP), veáse SÜCHTING-HÄNGER, Andrea: «Das Gewissen der Nation»: Nationales Engagement und politisches Handeln konservativer Frauenorganisationen 1900 bis 1917, Düsseldorf, 2002, pp. 325-326. 
El crítico año de 1932 proporciona un punto de partida para una visión de conjunto de los movimientos y culturas juveniles en la Alemania de entreguerras. Pero se analizará primero su evolución desde los inicios de la República de Weimar hasta la depresión, preguntándose cómo influyeron las nociones de «autonomía» de la juventud en la cultura del movimiento juvenil y de un espectro más amplio de organizaciones juveniles. A partir de este punto, se tratará el impacto de la depresión, cuando los defensores de un «Imperio de la juventud» protegido tuvieron que hacer frente a la aparente gran urgencia de los jóvenes por implicarse en la crisis política y económica. Incluso las connotaciones positivas asociadas con el aplazamiento de la edad adulta se pusieron en cuestión: surgió un nuevo modelo de hombre joven, visible en las marchas, desfiles, mítines y festivales, que era más vigorosamente «viril».

A partir de estos antecedentes de la historia de la «juventud organizada» de la República de Weimar, el artículo destacará tres cuestiones que han producido debate historiográfico. Todas ellas están relacionadas de diferente forma con la extensión de las continuidades y conexiones entre las organizaciones y culturas juveniles en la República de Weimar por una parte, y las existentes durante la época nazi, por otra, y en todos los casos es la evolución durante los años de la depresión, en torno a 1929, y no sólo los sucesos de 1933, la que es fundamental. Un importante centro de interés han sido las afinidades ideológicas - y puntos de fricción- entre el movimiento juvenil alemán en su fase bündisch y el nacionalsocialismo en los años anteriores a $1933^{13}$. La misma pregunta se ha planteado con relación a las organizaciones juveniles católicas y protestantes ${ }^{14}$. Una segunda área de controversia ha surgido en torno a los compromisos con el nazismo que buscaron algunas organizaciones como respuesta a la toma del poder por parte de los nazis ${ }^{15}$. Esto no se aplica, por supuesto, a las organizaciones juveniles de izquierda, a las que este debate sobre el compromiso no se les presentó: fueron las primeras en ser prohibidas junto con el resto del movimiento obrero socialista ${ }^{16}$. Pero el espacio de maniobra que les quedó a las organizacio-

13 REULECKE, Jürgen: “"Hat die Jugendbewegung den Nationalsozialismus vorbereitet?” Zum Umgang mit einer falschen Frage», en KrabBe, Wolfgang R. (dir.): Politische Jugend..., op. cit., pp. 222-243.

14 Götz Von Olenhusen, Irmtraud: Jugendreich, Gottesreich, Deutsches Reich: Junge Generation, Religion und Politik 1928-1933, Colonia, 1987; véase también HARvEY, Elizabeth: "Gender, generation and politics: young Protestant women in the final years of the Weimar Republic» en RosEMAN, Mark (dir.): Generations in Conflict. Youth Revolt and Generation Formation in Germany 17701968, Cambridge, 1995, pp. 184-209.

15 Hellfeld, Matthias von: Bündische Jugend und Hitlerjugend: Zur Geschichte von Anpassung und Widerstand 1930-1939, Colonia, 1987, pp. 73-98; comentarios sobre este debate se pueden ver también en ReULECKE, Jürgen: «Hat die Jugendbewegung...», op. cit. Véase además el informe recogido en GIESECKE, Hermann: Vom Wandervögel bis zur Hitlerjugend: Jugendarbeit zwischen Politik und Pädagogik, Munich, 1981, pp. 184-191.

16 EBERTS, Erich: Arbeiterjugend 1904-1945: Sozialistische Erziehungsgemeinschaft, Politische Organisation, Frankfurt, 1980, pp. 152-157; KLÖNNE, Arno: «Jugendprotest und Jugendopposition: 
nes no socialistas que no fueron ilegalizadas de forma inmediata fue menos claro, y sus respuestas a los movimientos hechos por las autoridades nazis fueron, en consecuencia, variadas. Este artículo se preguntará qué esperaban ganar compartiendo la suerte de la «revolución nacional» aquellas organizaciones que realmente optaron por un compromiso con el régimen en sus fases iniciales, y cual fue el resultado de esta estrategia. La tercera pregunta se relaciona con las consecuencias de la campaña nacionalsocialista para eliminar a todas las organizaciones juveniles no nazis y cómo su campaña por la conformidad desencadenó disidencia, oposición y resistencia juvenil. Los historiadores han luchado con el problema de caracterizar y evaluar la importancia de las diversas partes de esta oposición, que varió desde las actividades de resistencia de los jóvenes comunistas y socialistas y las acciones de protestantes y católicos en defensa de sus creencias y costumbres, a las manifestaciones espontáneas de desafío y de rebelión juvenil asociadas con las tradiciones bündisch y las subculturas proletarias. En particular, los historiadores han tenido como objetivo explorar qué similaridades y continuidades existieron entre las formas de expresión juvenil anteriores a 1933 y las formas de disidencia e inconformidad que surgieron durante el régimen nazi ${ }^{17}$.

\section{1. «IMPERIOS DE LA JUVENTUD»: LA «JUVENTUD ORGANIZADA» EN LA ALE- MANIA DE WEIMAR}

La noción de autonomía fue un elemento central en la formación del Wandervögel de preguerra, una red de grupos de excursionismo que florecieron entre los estudiantes alemanes y austríacos de los institutos de educación secundaria desde finales del siglo XIX y que destacaban frente a otras organizaciones de la época por su reivindicación de ser un movimiento de, más que para jóvenes ${ }^{18}$.

Dentro de su propio «imperio», las supuestas cualidades especiales de los jóvenes podían ser educadas con el objetivo de prepararlos para su misión de restaurar la «integridad» y la «autenticidad» a una sociedad desordenada. Las excursiones de grupo tenían como objetivo dar a los protegidos jóvenes urbanos un nuevo sentido de libertad física a través del ejercicio, la oportunidad de explorar el campo y la experiencia de camaradería dentro de un grupo de edad bajo la dirección de un líder elegido y escasamente mayor. El Wandervögel ofre-

Von der HJ-Erziehung zum Cliquenwesen der Kriegszeit», en BROsZAT, Martin, FRÖHLICH, Elke y Grossmann, Anton (dirs.): Bayern in der NS-Zeit IV: Herrschaft und Gesellschaft im Konflikt, Munich, 1981, pp. 527-620, esp. pp. 554-564.

17 Discusiones sobre los estudios existentes se pueden ver en BREYvoGel, Wilfried: «Jugendliche Widerstandsformen —vom organisierten Widerstand zur jugendlichen Alltagsopposition», en SteINBACH, Peter y TuCHel, Johannes (dirs.): Widerstand gegen den Nationalsozialismus, Berlín, 1994, pp. 426-442; KENKMANN, Alfons: Wilde Jugend. Lebenswelt großstädtischer Jugendlicher zwischen Weltwirtschaftskrise, Nationalsozialismus und Währungsreform, Essen, 1996, pp. 15-22.

18 LAQUeUR, Walter: Young Germany: A History of the German Youth Movement, Nueva York, 1962. 
cía una forma de escape de las convenciones sociales a la naturaleza y a la camaradería del grupo de edad. Se consideraba que la búsqueda de sus «propios valores» por los hombres jóvenes les implicaba, uniéndose con otros, en un mundo imaginado como liberación de la familia burguesa, y que específicamente excluía a chicas y mujeres ${ }^{19}$. Las jóvenes que querían unirse a estas organizaciones planteaban un problema a los defensores de una nueva masculinidad que debía lograrse a través de la unión de los hombres. Los grupos del Wandervögel se dividieron respecto de este asunto y las chicas fueron aceptadas en algunos grupos pero excluídas de otros ${ }^{20}$. Hacia 1913 el Wandervögel estaba establecido y ya dividido en facciones: no sólo la participación de las chicas, sino también la de los judíos estaban creando controversia. Mientras tanto, aunque la novedad de ir de excursión, cantar y dormir sobre paja todavía fascinaba a los nuevos miembros, otros buscaban empujar el movimiento en una nueva dirección. En este proceso, crearon nuevos lemas sobre el tema de la juventud y su misión en la sociedad. La Freideutsche Jugend (Juventud Libre Alemana) fue fundada como una nueva red para los miembros mayores del movimiento juvenil en el famoso mitin en la ladera del Hoher Meissner en octubre de 1913, un «contrafestival» organizado frente a la celebración oficial y jingoísta del centenario de la batalla de Leipzig. Una declaración aprobada en el mitin declaraba que la Freideutsche Jugend, se comprometía a «modelar su propia vida de acuerdo con su propia vocación, bajo su propia responsabilidad y con autenticidad interior». Repetida hasta la saciedad, la «declaración de Meissner»se convirtió en el estatuto del movimiento juvenil alemán ${ }^{21}$.

En los años veinte, la visión de un «Imperio de la juventud» (Jugendreich) continuó inspirando a la llamada Bündische Jugend, que continuaba la tradición del movimiento de preguerra en forma de unas Bünde organizadas más firmemente: enfatizaban las relaciones entre líderes y seguidores, la segregación por sexos y las estructuras internas basadas en la división por grupos de edad ${ }^{22}$. La idea de Jugendreich todavía tenía un potencial de emancipación. Para las chicas, por ejemplo, la comunidad del grupo de edad proporcionaba un contexto en el que podían experimentar —en grupos segregados por sexos- la libertad física y la aventura, una ruptura de la vida familiar, un sentido de pertenencia y un placer compartido por el excursionismo, los fuegos de campamento, las canciones folklóricas y el baile. Algunas mujeres jóvenes destacaban la experiencia de una nueva «hermandad femenina», mientras otras veían el espacio autónomo del movimiento juvenil como

19 ReULECKE, Jürgen: «Männerbund versus Familie: Bürgerliche Jugendbewegung und Familie in Deutschland im ersten Drittel des 20. Jahrhunderts», en KOEBNER, Thomas, JANZ, RolfPeter, y Trommler, Frank (dirs.): «Mit uns zieht die neue Zeit», op. cit., pp. 199-223, esp. pp. 203-204.

20 Sobre las chicas en el Wandervögel, véase el número monográfico de Jabrbuch des Archivs der Deutschen Jugendbewegung (Witzenhausen), 15 (5/1984).

21 REULECKE, Jürgen: «Männerbund versus Familie...», op.cit.

22 FIEDLER, Gudrun: Jugend im Krieg: Bürgerliche Jugendbewegung, Erster Weltkrieg und sozialer Wandel 1914-1923, Colonia, 1989, pp. 178-182. 
un lugar para debatir sobre las identidades femeninas ${ }^{23}$.

Mientras tanto, la idea de autonomía juvenil empezó a influir en un espectro más amplio de la juventud organizada. Antes de 1914, las organizaciones juveniles de las iglesias cristianas y las de los jóvenes trabajadores del movimiento socialdemócrata se habían desarrollado en gran medida siguiendo las líneas de la asistencia a los jóvenes tradicional, conocida como Jugendpflege y dirigida por adultos ${ }^{24}$. Sin embargo, después de la Primera Guerra Mundial, las distinciones entre «asistencia a los jóvenes» $\mathrm{y}$ «movimiento juvenil» se difuminaron: los elementos de autoorganización se extendieron de una forma más amplia ${ }^{25}$. En parte, esto se debió a las tendencias generales de democratización y movilización política de masas durante los primeros años de la República: tanto las chicas como los chicos eran ahora futuros ciudadanos de un Estado democrático y podían esperar tener derecho de voto al alcanzar los 20 años. Más aún, los intereses propios de todos los grupos que buscaban tener influencia en la política de Weimar — partidos políticos, sindicatos, iglesias, asociaciones paramilitares, grupos feministas- determinaron algunos de los movimientos que buscaban animar la participación juvenil ${ }^{26}$. Era una «verdad indiscutible» de la época que asegurar el futuro significaba reclutar a la joven generación y, a la vez, esto parecía requerir la creación de espacios para la participación juvenil. Sin embargo, la autonomía tenía sus límites y algunas organizaciones defendían poco más que de boquilla la idea de iniciativa juvenil ${ }^{27}$. Algunas secciones juveniles de partido tendían a ser vistas por los dirigentes de éstos como un simple depósito de votos para el futuro, y los militantes jóvenes de las secciones juveniles de partido de mentalidad más independiente - como la Windhorstsbunde del Zentrum, el partido católico- era probable que entraran en conflicto con las direcciones de sus respectivos partidos ${ }^{28}$.

El movimiento juvenil alemán del periodo anterior a la Primera Guerra Mundial había incluido elementos de rebelión cultural con su denuncia del mercantilismo, la cultura de masas y las convenciones burguesas. Algunos acti-

23 Una historia de las chicas y las mujeres jóvenes en las Bünde del periodo de Weimar se puede ver en DE RAS, Marion E. P.: Körper, Eros und weibliche Kultur: Mädchen im Wandervogel und in der Bündischen Jugend 1900-1933, Pfaffenweiler, 1988, pp. 100-239.

24 La sección juvenil del Partido Socialdemócrata había sido obligada a abandonar todo elemento de reivindicación política por la Ley Nacional de Asociaciones (Reichsvereinsgesetz) de 1908, que prohibía a los menores de 18 años toda actividad política: EBERTs, Erich: Arbeiterjugend..., op. cit., pp. 28-29. Sobre las organizaciones juveniles católicas y protestantes antes de la Primera Guerra Mundial, ver GÖTZ VON OlenHUSEN, Irmtraud: Jugendreich..., op. cit., pp. 65-66 y 129.

25 GIESECKE, Hermann: Vom Wandervogel bis zur Hitlerjugend..., op. cit., pp. 86-87; SPEITKAMP, Wilfried: Jugend in der Neuzeit: Deutschland vom 16. bis zum 20. Jahrbundert, Göttingen, 1998, pp. 183-184.

26 KRABBE, Wolfgang R.: «Einleitung», en Idem (dir.): Politische Jugend..., op. cit., p. 10.

27 KRABBE, Wolfgang R.: «Parteijugend in der Weimarer Republik: Ein typologischer Vergleich am Beispiel der Zentrums- und der DVP-Jugend', en Idem (dir.): Politische Jugend..., op. cit., pp. 38-72, p. 43.

28 Ibid. 
vistas del movimiento juvenil usaron sus experiencias dentro del movimiento como una plataforma para una reforma social de largo alcance. En el periodo que siguió a la Primera Guerra Mundial, antiguos miembros del movimiento juvenil estuvieron en la vanguardia de nuevos enfoques en la educación, el trabajo social y el tratamiento de la delincuencia juvenil ${ }^{29}$. Sin embargo, las cualidades radicales del movimiento juvenil fueron amortiguadas con el tiempo. Sus prácticas, desarrolladas como métodos de unión de grupos — la excursión, el campamento, el mitin- pronto se reprodujeron de forma rutinaria y fueron ampliamente imitadas y, hasta cierto punto, institucionalizadas, por ejemplo, con la creación de una red de albergues juveniles ${ }^{30}$. A finales de los años veinte, estas actividades llegaron a ser técnicas convencionales para «organizar» a los jóvenes y eran tratadas de forma benevolente por los políticos y los «expertos» en la juventud atraídos por la idea de promover la salud y la displina en la generación emergente y, en primer lugar entre los adolescentes de clase obrera, que se consideraba que necesitaban más protección y control ${ }^{31}$. Los «no organizados» empezaron a ser caracterizados como la categoría de jóvenes más «difícil» por políticos y pedagogos preocupados por el bienestar juvenil y la política social: era como si la decisión de tratar de evitar cualquier contacto con las formas organizadas de ocio fuera un indicador de una conducta «asocial» ${ }^{32}$.

Así pues, a lo largo de los años veinte, las organizaciones juveniles llegaron a proporcionar un ocio organizado a un amplio sector de alumnos de escuela, estudiantes, oficinistas en prácticas, aprendices y jóvenes trabajadores. En 1926, el Consejo Nacional de Organizaciones Juveniles llevó a cabo una encuesta basada en las organizaciones afiliadas a él ${ }^{33}$. Los resultados de esta encuesta indicaron que el número total de afiliados de entre 14 y 21 años era de 3,9 millones: teniendo en cuenta que algunos jóvenes eran miembros de más de una organización, se estimaba que en torno al 40 por ciento de los 9,1 millones de alemanes de entre 14 y 21 años formaba parte de una asociación juvenil ${ }^{34}$. Las organizaciones con mayor número de miembros en este grupo de edad eran las deportivas (1,6 millones en 1926, de los cuales 1,3 eran hombres) y las organizaciones de base confesional de las iglesias cristianas (784.000 en las orga-

29 HarveY, Elizabeth: Youth and the Welfare State..., op. cit., pp. 154-155.

30 GIESECKE, Hermann: Vom Wandervogel bis zur Hitlerjugend..., pp. 86-87.

31 Ibid., pp. 140-157.

32 KenKmann, Alfons: Wilde Jugend..., op. cit., pp. 75-76.

33 Se requería a las organizaciones asociadas al Consejo que jurasen lealtad a la República de Weimar, y que demostrasen que tenían al menos 50 grupos locales con un total de un mínimo de 1.000 afiliados entre los 14 y los 25 años y que su principal objetivo era fomentar el desarrollo físico, intelectual y moral de los jóvenes. Las organizaciones paramilitares, las comunistas y las nazis estaban excluídas. GIESECKE, Hermann: Vom Wandervögel bis zur Hitlerjugend..., op. cit., p. 159.

34 MEwes, Bernhard: Die erwerbstätige Jugend: Eine statistische Untersuchung, Berlín y Leipzig, 1929, pp. 151-159. 
nizaciones católicas y 458.950 en las protestantes) ${ }^{35}$. El número de afiliados de las agrupaciones clasificadas como Bündische Jugend era pequeño: se consideraba que pertenecían a ellas alrededor de 34.000 chicos y hombres jóvenes y 17.000 chicas y mujeres jóvenes en $1926^{36}$. Un cálculo adicional estimaba que unos 700.000 hombres jóvenes menores de 21 años eran miembros de secciones juveniles de organizaciones paramilitares que no formaban parte del Consejo Nacional de Organizaciones Juveniles ${ }^{37}$. Era más probable que estuvieran «organizados» los jóvenes que las jóvenes: alrededor de un tercio de los miembros de las organizaciones juveniles encuestadas en 1926 eran mujeres ${ }^{38}$. Un cálculo de 1928 sobre Prusia sugería que el $57 \%$ de los jóvenes y el $34 \%$ de las jóvenes estaban «organizados» ${ }^{39}$. Las actividades de estas asociaciones habían llegado a ser una señal de «adolescencia saludable» y un mecanismo para integrar a los jóvenes en un ámbito social particular: una gama de diferentes grupos adolescentes, cada uno con un sistema de valores e insignias particulares y que proporcionaban a sus miembros una red previsible y segura para realizar actividades de ocio —excursionismo, música, deporte- dentro del mundo tranquilizador de aquellos que tenían una mentalidad parecida. Los católicos podían hacer excursiones, o incluso jugar al fútbol, con otros católicos: una de las mayores organizaciones deportivas era la católica Deutsche Jugendkraft, que contaba con medio millón de hombres menores de 21 años en $1926^{40}$. Las organizaciones juveniles proporcionaban una oportunidad a jóvenes judíos, socialistas o nacionalistas protestantes para afirmar - y debatir — sus identidades culturales y sus actitudes políticas. Unirse a una organización juvenil podía, alternativamente, ser una cuestión de elección de consumo: la gente joven podía optar por aquella que ofrecía las actividades más atractivas y también más asequibles económicamente.

Imaginar un ámbito en el que los jóvenes estarían protegidos de las tentaciones del «materialismo» al igual que de la lucha política atraía a aquellos para quienes la evolución social y política de la época de Weimar era inquietante. Pero es difícil de reconstruir si esto significaba mucho para los militantes de base de estas organizaciones. Sin embargo, estaba asociado con un código de conducta - abstinencia, disciplina, camaradería o cuidado de la salud físicaque se inculcaba a sus miembros junto con los valores distintivos ligados a cada organización concreta. En el movimiento juvenil burgués y sus bünde prevalecía

35 Ibid., pp. 154-155.

36 Ibid.

37 Ibid., p. 158.

38 «Die Mädchen in den Jugendverbänden» en WolfF, Emmy (dir.): 11. Jahrbuch des Bund Deutscher Frauenvereine (1927-8), Mannheim, 1928, p. 81.

39 Las cifras sobre Prusia en 1928 están recogidas en Sonderheft: «Mädchenerziehung in den Jugendverbänden: Wandlungen, Zustände, Ziele», en Das Junge Deutschland (Berlín), vol. 24, nº. 9 (Septiembre 1930), pp. 389-481, p. 392.

40 GÖTZ VOn Olenhusen, Irmtraud: Jugendreich..., op. cit., p. 66; Mewes: Die erwerbstätige Jugend, op. cit., p 155. 
una actitud «nacional», aunque los matices seguían siendo importantes: un patriotismo de mentalidad liberal podía caracterizar a una Bund, el ultranacionalismo y antisemitismo a otra ${ }^{41}$. Los principios centrales de las organizaciones cristianas eran la piedad, la castidad y la obediencia a Dios junto a fuertes afirmaciones de nacionalidad alemana ${ }^{42}$. Las organizaciones judías — que iban desde la organización sionista Blau-Weiss fundada antes de la Primera Guerra Mundial a las organizaciones juveniles judías liberales que destacaban que formaban parte de la "patria alemana»_ compartían un compromiso para explorar y mantener su sentido de identidad judía y demostrar sus fuertes lazos con la naturaleza, frente a la asunción común que equiparaba a los judíos con la modernidad urbana ${ }^{43}$. El objetivo común de los jóvenes socialistas de la Sozialistische Arbeiterjugend (Juventud Obrera Socialista), era la creación de una humanidad nueva, que construiría el socialismo del futuro sobre las bases de la solidaridad de clase, la igualdad de género y el internacionalismo ${ }^{44}$. Estos mensajes se extendían entre los afiliados a través de mítines, y eran reforzados por boletines y periódicos diseñados para consolidar un sentido de peculiaridad tanto en comparación con otras organizaciones como con relación a los «no organizados», que generalmente eran considerados víctimas de la cultura de masas modernas y la superficialidad de la vida urbana.

\section{LA BÚSQUEDA DE UN COMPROMISO}

«Ya no hay una «tierra de la juventud» neutral» comentó — aparentemente con pena— Erich Ollenhauer, el líder de la Sozialistische Arbeiterjugend, en el congreso de su organización en Leipzig en 193145. Los años de la depresión fueron un momento decisivo en el desarrollo de la «juventud organizada» en la Alemania de entreguerras. La noción de un «imperio de la juventud» autónomo, como una zona de abstinencia y evasión, distinguida de las presiones políticas y de las realidades económicas, llegó a parecer cada vez más reñida con los tiempos, al ser arrastradas millones de personas a la pobreza mientras se des-

41 Sobre la política de la Bündische Jugend, ver LAQUEUR, Walter: Young Germany..., op. cit; HeLlFELD, Matthias von: Bündische Jugend und Hitlerjugend..., op. cit., pp. 33-48.

42 Sobre los ideales prescritos para los chicos y chicas católicos ver GÖTZ VON OLENHUSEN, Irmtraud: Jugendreich..., op. cit., pp. 59-88.

43 BRENNER, Michael: The Renaissance of Jewish Culture in Weimar Germany, New Haven y Londres, 1996, pp. 46-49.

44 EBERTS, Erich: Arbeiterjugend..., op. cit., pp. 70-76; sobre las chicas en la SAJ, ver Martina Naujoks, «Profile einer Minderheit: Mädchen in der Sozialistischen Arbeiterjugend», en Jabrbuch des Archivs der Deutschen Jugendbewegung vol. 15 (5/1984), pp. 137-152.

45 EBerTs, Erich: Arbeiterjugend..., op. cit., p. 107. Dos décadas más tarde, en 1952, Ollenhauer sucedería a Kurt Schumacher como líder del Partido Socialdemócrata de la República Federal de Alemania. 
truía la legitimidad del gobierno parlamentario. Los dirigentes de las organizaciones más «apolíticas» fueron presionados por sus afiliados para hablar de temas de actualidad y las secciones juveniles de los partidos políticos o de las organizaciones paramilitares demandaron una mayor participación en las decisiones y campañas. Al mismo tiempo, la sensación de crisis nacional hizo a los dirigentes juveniles tomar posiciones políticas.

En mayo de 1932, el ministro prusiano de Industria y Comercio habló de la necesidad de combatir la «catástrofe moral» que el desempleo de larga duración produciría en la juventud ${ }^{46}$. Parecía claro para los observadores contemporáneos que el impacto de la depresión sobre todas las categorías de jóvenes - chicos y chicas que acababan la escuela, trabajadores manuales, cualificados o no, y jóvenes universitarios que buscaban salidas profesionales - sería devastadora para las habilidades y la moral de trabajo y aumentaría el vagabundeo, el crimen, la prostitución y las filas de los políticamente desafectos ${ }^{47}$. En las ciudades industriales del Ruhr y en Berlín, el desempleo de larga duración proporcionó las bases para la extensión de las cliques, grupos o bandas informales de clase obrera, que generalmente se desarrollaban en los barrios proletarios y tenían fama de alterar el orden público y de conducta conflictiva ${ }^{48}$. Las percepciones contemporáneas de crisis y desorden se acentuaban aún más por el espectáculo de las formaciones paramilitares movilizadas en las calles, algunas veces en defensa de la República, pero mayoritariamente en contra de ella. Las organizaciones paramilitares —incluidas las antirrepublicanas de la izquierda comunista y de la derecha nacionalista radical (Stalbelm y SA)— ofrecían a algunos jóvenes desempleados un ambiente juvenil y una red de actividades ${ }^{49}$. La violencia política creció, particularmente en el crítico año de 1932, a pesar de la prohibición gubernamental de las organizaciones paramilitares antirrepublicanas, prohibición que se hacía cumplir más constantemente contra los comunistas que contra la extrema derecha. Los modelos de lealtad vecinal de los medios proletarios aumentaban la violencia en las calles. En Berlín, los miembros de las cliques eran, en ocasiones, cortejados por los comunistas en su campaña para defender los barrios obreros de las «incursiones» fascistas ${ }^{50}$. En Düsseldorf, por el contrario, miembros de la Wander-Bund Kittelbach-Piraten, una organización de jóvenes de clase obrera con fama de duros, simpatías extremistas de derecha y du-

46 HARVEY, Elizabeth: Youth and the Welfare State..., op. cit., p. 121.

47 Ibid., p. 127.

48 RosenHAFT, Eve: «Die wilden Cliquen», en Deutscher Werkbund e.V. y Württembergischer Kunstverein Stuttgart: Schock und Schöpfung: Jugendästhetik im 20. Jabrhundert, Darmstadt, 1986, pp. 345-349; ROSENHAFT, Eve: «Organizing the «Lumpenproletariat»: Cliques and Communists in Berlin during the Weimar Republic», en EvaNs, Richard (dir.): The German Working Class 1888-1933, Londres, 1982, pp. 174-220.

49 PeuKerT, Detlev: «Lost Generation»..., op. cit., pp. 188-189.

50 Rosenhaft, Eve: Beating the Fascists? The German Communists and Political Violence 19291933, Cambridge, 1983, pp. 131-138. 
raderas conexiones con varias Bünde paramilitares de los años 20, apoyaron a las SA en las luchas callejeras con los comunistas en $1932^{51}$.

Durante la crisis económica y política de los años de la depresión, las organizaciones juveniles —incluso aquellas que se habían declarado antes fundamentalmente «apolíticas»— participaron en la movilización de las fuerzas cada vez más polarizadas que buscaban preservar la república y su constitución, derrotarla en nombre de la revolución comunista o destruirla en busca de un «Tercer Reich». Fue un periodo en el que las fuerzas en conflicto en la política alemana trataron de atraer a la «juventud» y desarrollaron un estilo externo diseñado para transmitir preparación para «la lucha». Los niños y jóvenes de las organizaciones juveniles protestantes y católicas, de la juventud socialista y la comunista, y de las Bünde nacionalistas de derecha adoptaron cada vez más el estilo de «formaciones de combate», algunas veces enfrentándose unas con otras en la calle y en las excursiones de fin de semana al campo. Los uniformes y las insignias militares llegaron a estar muy de moda. Parecía, también, que las organizaciones de la Bündische Jugend estaban poniendo un nuevo énfasis en el ideal de masculinidad adulta, más que joven: «El descontento de la juventud ya no se dirige a modelar un imperio de la juventud: anhela tareas varoniles» escribió en 1932 Alfred Moritz, un representante de una de las Bünde nacionalistas, en el periódico citado al comienzo de este artículo ${ }^{52}$. Parece como si hubiera sido esencial para las organizaciones católicas rebatir cualquier impresión de que el mundo católico era de alguna forma débil o «feminizado». Se afianzó una forma de «Cristianismo fuerte», que ponía el énfasis en el Cristo victorioso más que en el Cristo doliente $e^{53}$. Aunque estas tendencias buscaran proporcionar un nuevo centro de interés e ímpetu para los niños y jóvenes «organizados», también servían para empujar más aún a las organizaciones de mujeres jóvenes «fuera del candelero». Esta evolución recalcaba el cambio de la política desde las asambleas parlamentarias a la calle, y la marginación cada vez mayor de las mujeres en la vida política de lo que quedaba de la República ${ }^{54}$.

Muchos dirigentes de organizaciones juveniles —socialistas, católicos, protestantes o bündisch - esperaban que la convulsión política tuviera resultados positivos. Imaginaban ser parte de un reordenamiento de la política y de la sociedad que concordaría con su conjunto de valores particular. Al continuar la crisis política, algunos portavoces de la Bündische Jugend creyeron que el rechazo cada vez mayor de los principios de la política liberal podía desarrollar cambios acordes con su forma de pensar. En general, los representantes de las Bünde

\footnotetext{
51 Kenkmann, Alfons: Wilde Jugend..., op. cit., pp. 59-63.

52 «Sonderheft: Jugendverbände in der Krise der Gegenwart, Erste Folge», op. cit., p. 130: aportación de Alfred Moritz, Freischar Junger Nation, 'Die Unruhe der Jugend gilt nicht mehr der Gestaltung eines Jugendreichs, sie sehrt sich nach männlichen Aufgaben’.

53 GÖTZ VON OlenHUSEN, Irmtraud: Jugendreich.., op. cit., pp. 80-96; RuFF, Mark: The Wayward Flock: Catholic Youth in Postwar West Germany, 1945-1965, Chapel Hill (NC), 2005, pp. 22-24.

54 SüCHTING-HÄNGER, Andrea: «Das Gewissen der Nation»..., op. cit., p. 325.
} 
habían sido reacios a participar en la política dentro del marco de la democracia de Weimar, alegando que ésta estaba plagada de presiones de los grupos de interés y de compromisos insatisfactorios. Estas características de la democracia liberal estaban reñidas con el ideal bündisch de una comunidad nacional «orgánica» (Volksgemeinschaft) basada en la reconciliación de las clases y en una división «natural» del trabajo entre los sexos. Ahora, sin embargo, diferentes miembros de la Bündische desafiaban el abstencionismo del movimiento juvenil al buscar importar las ideas de éste a la vida política ${ }^{55}$. Un resultado de estas ideas fue el experimento de corta duración que afectó al antiguo Partido Democrático Alemán (Deutsche Demokratische Partei, DDP), que unió sus fuerzas en 1930 con la Asociación Nacional Popular del Reich (Volksnationale Reichsvereinigung) formada por Arthur Mahraun y sus colegas de la Orden Joven Alemana (Jungdeutscher Orden), una formación bündisch hostil al sistema de partidos políticos existente pero declaradamente leal a la República como forma de Estado. El resultado de esta colaboración fue el nuevo Partido del Estado Alemán (Deutsche Staatspartei). Sin embargo, éste no fue capaz de servir como base para una unión mayor del republicanismo liberal y tampoco tuvo éxito en movilizar apoyo electoral, por lo que Mahraun pronto retiró su apoyo al proyecto ${ }^{56}$.

Otras iniciativas tomadas por la juventud organizada buscaban hacer frente a los efectos sociales de la crisis económica. El potencial de integración de las organizaciones juveniles, argumentaban sus líderes, podía tener relevancia para un amplio espectro de jóvenes «no organizados»: las asociaciones juveniles podían ofrecer modelos pedagógicos para hacer frente al desempleo juvenil masivo, y atraer así a los «no organizados» y combatir la alienación social. Las organizaciones juveniles crearon estructuras para atender a sus propios militantes desempleados, pero también buscaron llegar a jóvenes no organizados con sus soluciones pedagógicas a la privación económica. Un experimento de pedagogía social muy publicitado que incluía a los desempleados fue promovido por una de las principales Bünde, el Deutsche Freischar. Tras haber desarrollado en los años veinte la idea de los campos de trabajo, en los que los hombres y mujeres jóvenes de diferentes orígenes sociales podrían superar las divisiones de clase a través del trabajo conjunto, estableció durante la depresión campos para los desempleados ${ }^{57}$. Estos fueron un modelo para el esquema establecido posteriormente por el gobierno de derechas de Brüning en 1931, el Servicio de Trabajo Voluntario (Freiwilliger Arbeitsdienst) ${ }^{58}$. Un tema recurrente en las contribuciones a la serie «Las organizaciones juveniles en la crisis actual» mencionada

55 LAQUEUR, Walter: Young Germany..., op. cit., p. 149.

56 JONES, Larry Eugene: «Liberalism and the Challenge of the Younger Generation: The Young Liberal Struggle for a Reform of the Weimar Party System, 1928-1930», en KRABBE, Wolfgang R. (dir.): Politische Jugend..., op. cit., pp. 106-128.

57 DuDEK, Peter: Erziebung durch Arbeit: Arbeitslagerbewegung und Freiwilliger Arbeitsdienst 1920-1935, Opladen, 1988.

58 HARvEY, Elizabeth: Youth and the Welfare State..., op. cit., pp. 136-47. 
al comienzo de este artículo era la importancia del Servicio de Trabajo Voluntario como un proyecto para la juventud organizada. Otro lema de la época, con gran resonancia entre la Bündische Jugend y discutido más ampliamente dentro de las organizaciones juveniles confesionales, era la idea de un asentamiento rural, especialmente en las regiones del este del Reich. Se sugería que los desempleados de las ciudades podrían encontrar un nuevo «espacio vital» y una nueva forma de vida en las tierras fronterizas del este de Alemania ${ }^{59}$. Estas propuestas tenían pocas oportunidades de realización y pueden ser consideradas principalmente como sueños para escapar de las complejidades de la vida urbana moderna a un modo de existencia más simple, más «auténtico» —una «receta» con escaso significado para la masa de desempleados.

Pero las fantasías escapistas eran, en cierto modo, comprensibles. A pesar de los discursos optimistas sobre un nuevo papel y una nueva relevancia, las columnas en marcha de las organizaciones juveniles eran en algunos casos una fachada de fortaleza que ocultaba la pérdida de militantes hacia organizaciones rivales y más radicales. Dentro del campo socialista, la polarización y radicalización cada vez mayor de la política produjeron realineamientos a expensas de la Sozialistische Arbeiterjugend y favorecieron agrupamientos socialistas más radicales como la sección juvenil del Partido Obrero Socialista (Sozialistische Arbeiterpartei (SAP), una escisión de izquierdas del SPD ${ }^{60}$. Entre las organizaciones políticas juveniles no socialistas, las secciones juveniles del partido liberal de derechas y del conservador nacionalista estaban estancándose o hundiéndose con la depresión económica, pero el Windborstbünde del Zentrum permaneció activo ${ }^{61}$. Además, hay evidencias, impresionistas más que cuantitativas, de que a los nazis se unieron «conversos» de los grupos bündisch y confesionales, para quienes los lemas de la «unidad nacional», la «comunidad nacional» y el «liderazgo» tenían una antigua atracción ${ }^{62}$. Principalmente para la juventud protestante de clase media después de 1930 — aunque los jóvenes católicos no eran inmunes - las organizaciones nazis parecían prometer la variante de política nacionalista más radical y el liderazgo más dinámico ${ }^{63}$.

59 FISCHER, Josepha: «Neue Impulse?», en «Sonderheft: Jugendverbände in der Krise der Gegenwart: Dritte Folge», en Das Junge Deutschland (Berlín), vol. 26, nº 7 (julio 1932), pp. 212-216, esp. p. 216; NoHL, Herman: «Landbewegung, Osthilfe und die nationale Aufgabe der Pädagogik», en Die Erziehung 7, no. 2 (1931-2), pp. 65-76.

${ }^{60}$ EBERTS, Erich: Arbeiterjugend..., op. cit., p. 109. La sección juvenil del SAP se llamaba Sozialistischer Jugendverband (SJV).

61 Sobre las organizaciones juveniles del DNVP (la Bismarckjugend) y del Deutsche Volkspartei (Partido del Pueblo Alemán (DVP) (la Hindenburgbund), ver GÖTZ Von Olenhusen, Irmtraud: Jugendreich..., op. cit., pp. 44-49; sobre la Windhorstbunde, ver KRABBE: «Parteijugend», en Idem (dir.): Politische Jugend..., op. cit.

62 Algunos ejemplos de «conversos» bündisch antes de 1933 se pueden ver en HELLFELD, Matthias von: Bündische Jugend und Hitler-Jugend..., op. cit., p. 53.

63 HARveY, Elizabeth: «Gender, generation and politics: young Protestant women in the final years of the Weimar Republic», en Roseman, Mark (Dir.): Generations in Conflict..., op. cit., pp. 184-209, esp. p. 205. 


\section{EL NAZISMO Y EL CULTO A LA CONFORMIDAD}

La «revolución nazi» supuso un dilema, aunque por poco tiempo, para los dirigentes de las organizaciones juveniles que no fueron disueltas por los nacionalsocialistas por ser parte de la izquierda. Al principio, los líderes de las formaciones nacionalistas de la Bündische Jugend y de organizaciones protestantes de similar orientación nacionalista creyeron que la nueva situación política les permitiría, al menos, influir más allá de los límites de sus organizaciones y llegar a la masa de jóvenes que sería enrolada en la Juventud Hitleriana. Estos fueron los motivos por los que los dirigentes juveniles bündisch y protestantes se embarcaron, durante 1933, en fatídicos compromisos, adaptándose fácilmente a las nuevas condiciones y ordenando que todas las cohortes de edad y/o las organizaciones en su conjunto se pasaran abiertamente a la Juventud Hitleriana, donde esperaban que podrían actuar como la «levadura» de una organización en crecimiento y proporcionar liderazgo a ésta ${ }^{64}$. Aquellos que permanecieron al margen de este curso de acción tuvieron la amarga satisfacción de que se demostrase, más tarde, que tenían razón cuando el régimen tomó medidas enérgicas contra lo que veía como evidencia de que los viejos estilos del movimiento juvenil se hacían sentir dentro de las organizaciones juveniles nazis ${ }^{65}$.

El movimiento nacionalsocialista afirmaba representar la voluntad política de una «joven generación» a la vez que subordinaba firmemente a los jóvenes a los dictados del régimen. Los dirigentes jóvenes nazis usaron la retórica de la autonomía juvenil, de la «juventud dirigida por la juventud», y adoptaron elementos del estilo y repertorio de las organizaciones juveniles preexistentes, a la vez que los convirtieron en instrumentos de la política ${ }^{66}$. Pero el culto a la juventud del nazismo enmascaraba el grado en que las energías de los jóvenes estaban siendo utilizadas para la guerra y la expansión territorial. Una vez que el nacionalsocialismo estableció su dictadura ya no tenía, como Kenkmann ha planteado, «necesidad de un ala juvenil activista o combativa» ${ }^{67}$. Lo que el régimen quería era gente joven que respondiera a las necesidades del Estado, que obedeciera órdenes y que fuera físicamente capaz de cumplir sus obligaciones hacia el pueblo $(V o l k)$. De acuerdo con esto, los jóvenes fueron organizados en las diferentes secciones de la Juventud Hitleriana, diseñadas para incorporar chicas y chicos en una «comunidad nacional» racialmente definida, y fueron sometidos a un programa intensivo de deporte y formación física, marchas y adoctrinamiento político. Surgió un grupo numeroso de otras instituciones y

64 HeLlfeld, Matthias von: Bündische Jugend und Hitler Jugend..., op. cit., pp. 80-98.

65 Ibid., pp. 99-118.

66 DomANSKY, Elisabeth: «Politische Dimensionen von Jugendprotest und Generationenkonflikt in der Zwischenkriegszeit in Deutschland», en Dowe, Dieter (dir.): Jugendprotest und Generationenkonflikt in Europa im 20. Jabrbundert: Deutschland, England, Frankreich und Italien im Vergleich, Bonn, 1986, pp. 113-137, pp. 135-136.

67 Kenkmann, Alfons: Wilde Jugend..., p. 65. 
formas de «servicio»— por ejemplo, el trabajo anual en granjas (Landjahr), para los jóvenes de clase obrera que terminaban el colegio, o el Servicio de Trabajo - como canales adicionales de entrenamiento y disciplina ${ }^{68}$. Los miembros de la Juventud Hitleriana, la Liga de Muchachas Alemanas y los participantes en el Landjahr y el Servicio de Trabajo eran enviados a campos que incluían entrenamiento deportivo o trabajo manual y en los cuales las presiones para ajustarse a la vida comunitaria nazificada se intensificaban mediante un programa agotador y la falta de privacidad ${ }^{69}$. Las iniciativas juveniles de la Juventud Hitleriana fueron canalizadas hacia la persecución de manifestaciones de «desviación» y subversión dentro de las filas de la organización y entre los jóvenes en general $^{70}$.

A comienzos de 1933, la Juventud Hitleriana y la Liga de Muchachas Alemanas - a pesar de todo su rápido crecimiento durante el crítico año de 1932 - todavía eran superadas en afiliados por otras organizaciones juveniles, sobre todo por las organizaciones de masas de la juventud católica y protestante. A finales de 1932, según los datos de la misma Juventud Hitleriana, tenía una militancia total de en torno a 108.000 afiliados (alrededor de 84.000 chi-

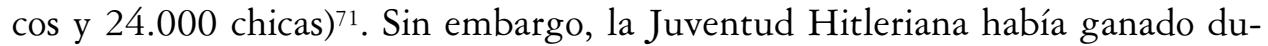
rante el periodo anterior a 1933 un perfil distintivo a través de sus polémicas estridentes, su imagen de revolucionarismo social, su enérgica agitación callejera en nombre de la causa nazi y, también, a través de sus «mártires», como Herbert Norkus, asesinado cuando distribuía panfletos en Berlín en enero de $1932^{72}$. El crecimiento de la Juventud Hitleriana tras la toma del poder por parte de los nazis — a finales de 1933 afirmaba haber alcanzado una militancia total de 2,3 millones - estuvo ligado a las medidas del régimen para destruir a sus rivales. Al consolidar el partido nazi su control del poder, la Juventud Hitleriana fue confirmada, en junio de 1933, como la «organización juvenil del Estado». En ese momento, las organizaciones juveniles socialdemócrata y comunista ya habían sido conducidas a la clandestinidad. El Líder Joven del Reich Alemán, Baldur von Schirach, se puso entonces a socavar a las organizaciones juveniles que quedaban. Una organización unitaria bastante improvisada en la que, en marzo 1933, se habían unido varias importantes Bünde nacio-

68 GIESECKE, Hermann: Vom Wandervögel bis zur Hitlerjugend..., op. cit., pp. 192-198.

69 SCHIEDECK, Jürgen y STAHLMANN, Martin: «Totalizing of Experience: Educational Camps», en SÜNKer, Heinz y OTTO, Hans-Uwe (Dir.): Education and Fascism: Political Identity and Social Education in Nazi Germany, Londres, 1997, pp. 54-80.

70 Sobre el establecimiento del llamado Streifendienst of the Hitler Youth (las patrullas de control de la HJ) en 1934 véase REMPEL, Gerhard: Hitler's Children. The Hitler Youth and the SS, Chapel Hill y Londres, 1989, pp. 52-56.

71 Hellfeld, Matthias von y KlÖNNE, Arno: Die betrogene Generation. Jugend im Faschismus, Colonia, 1987, p. 35.

72 Kenkmann, Alfons: Wilde Jugend..., op. cit., pp. 64-65; Hellfeld, Matthias von: Bündische Jugend und Hitlerjugend..., p. 51, ROSENHAFT: Beating the Fascists?..., op. cit., p. 143. 
nalistas no sirvió de protección, y Schirach la prohibió cuando llegó al poder en junio de $1933^{73}$. El Consejo Nacional de Organizaciones Juveniles fue disuelto en julio de ese $a \tilde{n} 0^{74}$. Las expresiones de lealtad no supusieron ninguna diferencia. Los líderes de las organizaciones juveniles protestantes hicieron lo posible para dar la bienvenida al nuevo régimen, pero en diciembre de 1933 sus organizaciones recibieron un ultimátum para que disolviesen sus agrupaciones de afiliados comprendidos entre los 10 y los 18 años o las integrasen en la sección correspondiente de la Juventud Hitleriana ${ }^{75}$. A partir de ese momento, el trabajo juvenil protestante quedó restringido a la asistencia pastoral de base parroquial. Desde enero de 1934, las únicas organizaciones juveniles de tamaño significativo permitidas fuera de la Juventud Hitleriana fueron las asociaciones católicas, que continuaron existiendo de momento debido al concordato firmado por Hitler con el Vaticano en julio de 1933. Durante los siguientes tres o cuatro años estas organizaciones hicieron frente a restricciones y acosos cada vez mayores, incluyendo prohibiciones regionales de organizaciones concretas. Finalmente, después de la confirmación oficial del monopolio de la Juventud Hitleriana por medio de la Ley de la Juventud Hitleriana de 1 de diciembre de 1936, todas las organizaciones fueron obligadas a desaparecer hacia 1937-1938 76 . En ese momento, la instrucción religiosa en el ámbito local parroquial fue la única forma de trabajo juvenil aún permitida a las dos confesiones cristianas fuera del marco de la Juventud Hitleriana.

Se permitió continuar existiendo después de 1933 a las organizaciones juveniles judías, aunque bajo grandes restricciones y una molesta vigilancia ${ }^{77}$. Sin embargo, su militancia floreció durante un tiempo, dado que los judíos que habían pertenecido a otras organizaciones juveniles ahora prohibidas se unieron a grupos específicamente judíos. Algunas asociaciones utilizaron esta oportunidad para entrenar a los jóvenes judíos en técnicas agrícolas como forma de prepararles para la emigración a Palestina ${ }^{78}$. En 1938, todos los grupos juveniles judíos fueron disueltos excepto el grupo sionista Hashomer Hazair.

Pero la Juventud Hitleriana no lo arrolló todo, a pesar de las imágenes de entusiasmo de masas propagadas por el régimen. Durante un corto periodo, la imagen antiburguesa de la Juventud Hitleriana fascinó a algunos jóvenes de

73 Hellfeld, Matthias von: Bündische Jugend und Hitlerjugend..., op. cit., pp. 95-96.

74 GIESECKE, Hermann: Vom Wandervogel bis zur Hitlerjugend..., op. cit., p. 184.

75 Hellfeld, Matthias von: Bündische Jugend und Hitlerjugend..., op. cit., p. 79; KLÖNNE, Arno, «Jugendprotest und Jugendopposition...», op. cit., p. 534.

76 KLÖNNE, Arno: «Jugendprotest und Jugendopposition...», op. cit., pp. 534-35; KLEINÖDER, Evi: «Verfolgung und Widerstand der Katholischen Jugendvereine», en BROSZAT, Martin y FRÖHLICH, Elke: Bayern in der NS-Zeit II, Munich, 1979, pp. 175-236.

77 Sobre las organizaciones judías después de 1933, véase PaucKer, Arnold: Deutsche Juden im Widerstand 1933-1945: Tatsachen und Probleme, Berlín, 1999, pp. 13-16.

78 Ibid., p. 61; ROGGE-GAU, Sylvia: «Widerstand von Juden im Alltag und in nationalsozialistischen Lagern», en STEINBACH y TUCHEL (dir.): Widerstand gegen den Nationalsozialismus..., op. cit., pp. 513-525, p. 515. 
clase media inspirados en la idea de una comunidad nacional sin clases, y su actitud desafiante hacia las figuras de autoridad tradicionales atrajo a aquellos que querían afirmarse frente a sacerdotes, profesores o padres ${ }^{79}$. La Juventud Hitleriana podía ofrecer la oportunidad de una carrera y una movilidad social ascendente a algunos chicos y chicas de origen obrero, mientras que sus competiciones deportivas o profesionales daban a otros su primera oportunidad de viajar lejos de ca$\mathrm{sa}^{80}$. Sin embargo, el gran crecimiento en el número de militantes de todas las secciones de la Juventud Hitleriana durante los primeros años del régimen se produjo en gran medida sobre la base de la incorporación forzada de las organizaciones que no eran nacionalsocialistas, y porque llegó a ser imposible realizar ninguna forma de deporte de esparcimiento físico fuera de ella. También se aplicaron diferentes formas de presión a potenciales nuevos reclutas incluso antes de la imposición formal de la militancia obligatoria. Los estudiantes de secundaria de clase media eran los más susceptibles a tales presiones, mientras que era más difícil presionar a los trabajadores jóvenes (mujeres y hombres) que habían dejado la escuela a los catorce años - con menos que perder en términos de perspectivas profesionales y estatus y con menos tiempo libre para actividades organizadas ${ }^{81}$.

A finales de los años treinta, la militancia de todas las secciones de la Juventud Hitleriana estaba alcanzando un punto de saturación, pero la organización había ya pasado el cenit de su atracción. La sección de la Juventud Hitleriana para chicos de 14 a 18 años (también llamada Hitler-Jugend o $H J$, presumiblemente para reflejar su status como «núcleo» o «vanguardia») se debilitó principalmente cuando muchos de sus dirigentes más competentes y comprometidos empezaron a ser reclutados para el Servicio de Trabajo y el Ejército (la Wehrmacht). Tanto entre las organizaciones de chicos como de chicas aumentaron las quejas por los ejercicios cada vez más numerosos y la rutina y la burocracia cada vez mayores ${ }^{82}$.

Mientras tanto, la cultura de la conformidad y las medidas enérgicas del régimen contra violaciones menores por parte de los jóvenes de la prohibición de reuniones o actividades «no oficiales» provocaron, no de forma sorprendente, una amplia variedad de respuestas desafiantes. Pero clasificar y analizar estas respuestas plantea dificultades al historiador. La cultura inconformista y los gestos de desafío deben ser distinguidos tanto en su intención como en su alcance de las acciones de los grupos de resistencia juvenil ligados normalmente a las organizaciones clandestinas de la izquierda ${ }^{83}$.

79 KLÖNNE, Arno: «Jugendprotest und Jugendopposition...», op. cit., p. 537.

80 ZimmermanN, Michael: «Ausbruchshoffnungen: Junge Bergleute in den dreïssiger Jahren», en NieThammer, Lutz (dir.): «Die Jabre weiss man nicht, wo man die heute hinsetzen soll»: FaschismusErfabrungen im Rubrgebiet, Berlín y Bonn, 1983, pp. 97-132, esp. pp. 100-101.

81 Kenkmann, Alfons: Wilde Jugend..., op. cit., p. 67.

82 KLÖNNE, Arno: «Jugendprotest und Jugendopposition»..., op. cit., pp. 544-545.

83 Sobre las actividades de resistencia de la organización juvenil comunista y de otras organizaciones juveniles de izquierda después de 1933, véase PEUKERT, Detlev: Die KPD im Widerstand: 
Sin embargo, algunas veces existieron continuidades y conexiones entre la inconformidad en la vida cotidiana, la determinación conciente de parte de la gente joven de desafiar las «demandas totalitarias» del régimen y afirmar su autonomía de diferentes formas, y la resistencia abierta. La elasticidad de las redes de amistad y las identidades y las costumbres de grupo formadas antes de 1933 - tanto en el ámbito bündisch como en el cristiano, judío o proletarioayudaron a mantener diferentes niveles de rechazo, rebelión y resistencia. De esta manera, los esfuerzos ilegales de los jóvenes para escapar al campo en excursiones y actividades de camping prohibidas, o para divulgar sus canciones y libros prohibidos, podían significar sobre todo un refugio mental y un «descanso» de la constante intromisión del régimen. Alternativamente, podían constituir un gesto de desafío antinazi política o religiosamente motivado. Podían, incluso, proporcionar una tapadera para que se reunieran miembros de las células clandestinas de resistencia. Hubo, por ejemplo, jóvenes comunistas activos en la resistencia en Berlín que utilizaron viejas redes de amistad, que se retrotraían a grupos de jóvenes judíos de los años veinte, para acumular apoyo para la resistencia dentro del frágil pero todavía legal refugio de las organizaciones juveniles judías. Después de que éstas fueran disueltas, un grupo simpatizante del comunismo se fusionó, en 1938-1939, con una célula de resistencia judía, cuyas actividades iniciales incluían las excursiones y los grupos de lectura y de música ${ }^{84}$. Por tanto, lo que parecía un grupo excursionista informal, podía tener todo, o nada, que ver con la resistencia política.

Las múltiples posibilidades asociadas con todos los agrupamientos informales de jóvenes y sus actividades estimularon las sospechas de la policía y pusieron en riesgo de ser perseguidos incluso a los más suaves intentos de incumplir las normas nazis, en especial si parecía que estaban ligados a la influencia bündisch. Varias Bünde se autodisolvieron antes que unirse a la nueva «juventud del Estado» en 1933: algunos de sus miembros continuaron sus anteriores actividades en secreto, algunas veces con nuevas variantes que surgieron de contactos con otros grupos prohibidos o todavía legales ${ }^{85}$. Muchos de los antiguos activistas juveniles que se unieron a la Juventud Hitleriana trataron de introducir en la organización las costumbres de las antiguas Bünde: una importación bündisch bastante obvia fue la tienda de campaña en forma de tipi indio, asociada en los últimos años veinte con un grupo conocido como el dj.1.11 y con su carismático líder Eberhard Köbel, también conocido como «Colmillo» ${ }^{86}$. La Juventud Hitleriana trató de acabar con tal «infiltración» y se las arregló para

Verfolgung und Untergrundarbeit an Rhein und Rubr 1933 bis 1945, Wuppertal, 1980, pp. 105, 142 y 304; KLÖNNE, Arno: «Jugendprotest und Jugendopposition...», op. cit., pp. 554-564.

84 Este era el grupo de Herbert Baum (véase KWIET, Konrad y EschWEGE, Helmut: Selbstbehauptung und Widerstand: Deutsche Juden im Kampf um Existenz und Menschenwürde 1933-1945, Hamburgo, 1986, pp. 114-139, especialmente p. 117).

85 HellFeld, Matthias von: Bündische Jugend und Hitlerjugend..., op. cit., pp. 119-145.

86 Ibid., pp. 101-106. 
deshacerse de varios exdirigentes bündisch que habían logrado puestos de dirección en la Juventud Popular alemana (Deutsches Jungvolk), la organización para chicos de entre 10 y 14 años $^{87}$. Para hacer frente a grupos de jóvenes ilegales considerados bündisch, el Ministerio del Interior aprobó un decreto, el 4 de febrero de 1936, que prohibía formalmente no sólo una serie de Bünde identificadas, sino también toda actividad bündisch: esta medida se añadió a una oleada de decretos ya aprobados para perseguir a las «cliques ilegales» y a los grupos juveniles definidos por el régimen como problemáticos ${ }^{88}$.

Mientras el régimen estaba haciendo sus primeros movimientos para eliminar a las Bünde y su influencia, las principales asociaciones juveniles católicas - acosadas y denigradas por el régimen pero autorizadas a continuar existiendo durante unos años después de la disolución forzada de las organizaciones juveniles protestantes — crecieron mucho durante el breve periodo comprendido entre 1933 y $1935^{89}$. Esto sucedió a pesar de las restricciones que les impuso el régimen, por ejemplo, la prohibición de toda actividad deportiva y la confiscación de oficinas e instalaciones. Los jóvenes que buscaban una alternativa a la Juventud Hitleriana se unieron a los grupos acosados y la tirada del periódico juvenil católico Junge Front se disparó de 85.000 ejemplares en julio de 1933 a 200.000 en junio de 1934 . El periódico, que recibió el nuevo nombre de $M i$ chael, finalmente fue prohibido en 1936. A pesar de todas las prohibiciones y restricciones a la actividad católica después de 1933, el hecho de que sus organizaciones juveniles disfrutaran de un periodo de desarrollo antes de ser disueltas a finales de los años 30 les dio tiempo a adaptarse a la creciente represión y a establecer un trabajo juvenil de base parroquial. Posteriormente, esto proporcionó una base tanto para reuniones legales con motivo de fiestas religiosas como para actividades ilegales, como el excursionismo o, en una más rara manifestación de abierta resistencia documentada en Eichstätt (Baviera), la producción secreta de folletos contra el régimen por un grupo de chicas católicas en cooperación con el capellán de la catedral local ${ }^{90}$.

Otras formas de rebelión y rechazo pueden remontarse a las cliques que proliferaron en las barriadas de clase obrera en los años veinte pero principalmente durante la depresión: en este caso era la cultura más sediciosa de la juventud «no organizada» la que proporcionaba un baluarte contra las demandas del régimen. Después de 1933, las bandas informales de jóvenes trabajadores industriales fueron un foco de hostilidad y agresión que tenía como objetivo a la Juventud Hitleriana. En las ciudades industriales del Rhin y del Ruhr surgieron grupos como los Kittelbach Pirates, que recordaban al Wanderbund Kittelbach-

87 Ibid., pp. 111-116.

88 KenKmanN, Alfons: Wilde Jugend..., op. cit., pp. 111-113.

89 KLÖNNE, Arno: «Jugendprotest und Jugendopposition...», op. cit., pp. 573-577; KLEINÖDER, Evi: «Verfolgung und Widerstand der katholischen Jugendvereine», op. cit., pp. 205-208.

90 KLEINÖDER, Evi: «Verfolgung und Widerstand der katholischen Jugendvereine», op. cit., p. 230. 
Piraten de los años veinte y de los años de la depresión, y los Navajos: algunas veces estos grupos se defínían a ellos mismos como tales, otras veces, eran las autoridades las que les daban estas denominaciones ${ }^{91}$. Tenían en común una propensión a la confrontación con la Juventud Hitleriana. El estilo y las actividades de estas cliques tomaba prestado, pero también parodiaba, los de las más respetables organizaciones juveniles del periodo de Weimar: los Kittelbach Pirates y los Navajos también iban de excursión, pero llevaban con ellos alcohol, cigarrillos y chicas, y cantaban canciones populares procedentes tanto del movimiento juvenil como del cine ${ }^{92}$. También adoptaban ropas e insignias particulares, pero con efectos extravagantes: pantalones cortos usados de forma provocadoramente corta y adornados con cremalleras, sombreros decorados con insignias y plumas al estilo tirolés, y calcetines blancos ${ }^{93}$.

El carácter ambiguo de estas cliques proletarias hacía posible que fueran interpretadas por las autoridades nazis de formas diferentes. La policía y los tribunales los veían algunas veces como delincuentes juveniles, y otras veces, como políticamente subversivos. No dispuesto o incapaz de distinguir entre los grupos excursionistas ilegales formados por jovenes trabajadores de aquellos de la prohibida Bündische Jugend, el régimen empezó a usar bündisch como una etiqueta que englobaba todas las formas de actividad juvenil ilegales y «desviadas». Era cierto que las cliques proletarias incorporaban elementos de la vida o la parafernalia bündisch en su propia subcultura para ser provocativos; sin embargo, el errático y elástico uso de esta denominación por parte del régimen es también un indicador de sus dificultades para luchar contra el fenómeno ${ }^{94}$.

\section{CONCLUSIONES}

La República de Weimar fue la gran era de la «juventud organizada», un periodo en el que una gran variedad de organizaciones, algunas selectas y elitistas, otras adaptadas a la militancia de masas, alcanzaron un gran número de afiliados. La idea de que las organizaciones juveniles podían crear un «Imperio de la juventud» autónomo para sus miembros fue ampliamente compartida. En diferentes grados, estas organizaciones y secciones juveniles reflejaban la visión de que los adolescentes y los adultos jóvenes estaban en una fase distintiva de la vida, gobernada por sus "propias leyes y valores», incluyendo un conjunto de normas de conducta bastante nobles y ascéticas, que eran condiciones previas para una transición saludable a la edad adulta. Estos valores pueden ser vistos como una base para una crítica radical a quienes prevalecían socialmente; pero

\footnotetext{
91 Ibid., pp. 75-79.

92 Ibid., p. 81.

93 Ibid., pp. 96-101.

94 Ibid., pp. 153-163.
} 
pueden también ser considerados como representación de una respuesta defensiva y preocupada a la modernidad.

La crisis económica y política de los años 1930 a 1933 tuvo consecuencias en muchas organizaciones y produjo algunos cuestionamientos a certidumbres previas relacionadas con el papel de los jóvenes en la sociedad. El impulso por «estar al día» dio lugar a algunos experimentos destinados al fracaso y también a algunos intentos más exitosos de intervenir en el ámbito político en busca de bienestar y trabajo para los jóvenes desempleados. Hacer frente a la turbulencia y polarización de la política significó también una adopción casi universal, compartida por los chicos y jóvenes «organizados», de los símbolos de la masculinidad militar. Las afinidades de estilo entre las organizaciones paramilitares de la extrema derecha y el nacionalismo firme y el «estilo de combate» de las formaciones de jóvenes católicos y bündisch ayudaron a engañar a los dirigentes juveniles, que creyeron que estaban destinados a ser parte de una política emergente de nacionalismo autoritario - un nuevo Reich - que parecía ser, de alguna forma, el resultado inevitable de la crisis política. Al final, por supuesto, sus intentos de alinearse con las masas en la esperanza de influirlas o incluso liderarlas fueron muy mal calculados.

Bajo el dominio nazi, el variado espectro de organizaciones juveniles del periodo anterior a 1933 desapareció. De las ruinas de las organizaciones juveniles anteriores a 1933 surgieron algunas fuentes significativas de resistencia antifascista, sostenidas por los estrechos lazos de amistad forjados entre los miembros de las organizaciones juveniles de izquierda y los grupos de jóvenes judíos antes de 1933. En las asociaciones de la Bündische Jugend y de la juventud protestante hubo desde un principio tanto un potencial de adaptación al régimen nazi como de rechazo y oposición a él. Las organizaciones juveniles católicas se adaptaron a las condiciones establecidas por el concordato, pero pronto fueron empujadas a una postura de desafío frente al acoso que culminó obligándolas a desaparecer hacia finales de los años 30 . Aunque sus acciones raramente llegaron a la resistencia abierta, muchos antiguos miembros de las organizaciones juveniles corrieron un riesgo considerable para mantener las redes de amistad y obtener formas de escape y alejamiento de las demandas del régimen nazi. Mientras tanto, la inconformidad juvenil «no organizada» adoptó el aspecto y los rituales del movimiento juvenil prohibido. Las excursiones se desarrollaron con un nuevo estilo y se extendieron a nuevos entornos sociales, cuando grupos variopintos de jóvenes de clase obrera se vistieron con pantalones cortos, camisas de tartán y sombreros tiroleses y se dirigieron a los campos y colinas. No hubo una línea más directa entre el Wandervögel y la cultura de inconformidad juvenil bajo el nazismo que la que hubo entre el $W$ andervögel y la Juventud Hitleriana. Pero todavía resulta asombroso cómo se volvió a poner de moda la costumbre de las excursiones como símbolo de libertad y autonomía juvenil — dirigida, ahora, contra las columnas en marcha de la Juventud Hitleriana.

(Traducción del inglés de Sandra Souto Kustrín). 